SUBJECT AREAS: MATERIALS SCIENCE NANOSCIENCE AND TECHNOLOGY

Received 10 July 2013

Accepted 15 November 2013

Published 5 December 2013

Correspondence and requests for materials should be addressed to D.H.W. (wangdh@nju. edu.cn) or Y.Y. (malab@nju.edu.cn)

\section{Excellent microwave absorption property of Graphene-coated Fe nanocomposites}

\author{
Xingchen Zhao', Zhengming Zhang' ', Liaoyu Wang' , Kai Xi' ${ }^{2}$, Qingqi Cao', Dunhui Wang' ${ }^{1}$ Yi Yang ${ }^{3}$ \\ \& Youwei Du'
}

\begin{abstract}
${ }^{1}$ Nanjing National Laboratory of Microstructures and Jiangsu Provincial laboratory for Nanotechnology, Department of Physics, Nanjing University, Nanjing 210093 , P. R. China, ${ }^{2}$ Nanjing National Laboratory of Microstructures and State Key Laboratory of Coordination Chemistry, Department of Polymer Science and Engineering, Nanjing University, Nanjing 210093, P. R. China, ${ }^{3}$ Jiangsu Provincial Key Laboratory of Photonic and Electronic Materials Sciences and Technology, School of Electronic Science and Engineering, Nanjing University, Nanjing 210093, P. R. China.
\end{abstract}

Graphene has evoked extensive interests for its abundant physical properties and potential applications. It is reported that the interfacial electronic interaction between metal and graphene would give rise to charge transfer and change the electronic properties of graphene, leading to some novel electrical and magnetic properties in metal-graphene heterostructure. In addition, large specific surface area, low density and high chemical stability make graphene act as an ideal coating material. Taking full advantage of the aforementioned features of graphene, we synthesized graphene-coated Fe nanocomposites for the first time and investigated their microwave absorption properties. Due to the charge transfer at Fe-graphene interface in $\mathrm{Fe} / \mathrm{G}$, the nanocomposites show distinct dielectric properties, which result in excellent microwave absorption performance in a wide frequency range. This work provides a novel approach for exploring high-performance microwave absorption material as well as expands the application field of graphene-based materials.

M icrowave absorbing materials (MAMs) are a kind of functional materials which can dissipate electromagnetic (EM) wave by converting it into thermal energy ${ }^{1}$. They have attracted more and more attention due to the important applications in microwave technology and radar detection ${ }^{1}$. Nanoparticles of magnetic metals are the potential MAMs for their high Snoek limit and reduced eddy-current losses ${ }^{2}$. However, these nanoparticles are apt to agglomerate and have strong affinity to oxide, which greatly limit their applications. To solve these problems, some oxides-coated magnetic metals nanocomposites, such as $\mathrm{Fe} / \mathrm{SiO}_{2}$, $\mathrm{FeNi}_{3} / \mathrm{SiO}_{2}, \mathrm{Fe} / \mathrm{Al}_{2} \mathrm{O}_{3}$, and $\mathrm{Fe} / \mathrm{ZnO}$, are synthesized, in which enhanced microwave absorption properties are observed $^{2-6}$. As we know, the microwave absorption capacity is mainly determined by the relative permittivity $\left(\varepsilon_{r}\right.$ $\left.=\varepsilon^{\prime}-i \varepsilon^{\prime \prime}\right)$, the relative permeability $\left(\mu_{r}=\mu^{\prime}-i \mu^{\prime \prime}\right)$, the EM impedance match, and the microstructure of the absorber ${ }^{2}$. When a beam of microwave irradiates the surface of an absorber, a good matching condition of the EM impedance can enable almost zero reflectivity of the incident microwave. And then, the transmitted microwave can be dissipated by dielectric loss and magnetic loss ${ }^{1}$. In the aforementioned coated nanocomposites, the real part $\left(\varepsilon^{\prime}\right)$ of relative permittivity keeps almost unchanged with the variation of microwave frequency, leading to the improper match of EM impedance and a relatively narrow absorbing frequency range ${ }^{2-4}$. In addition, as the coating layers, the density of $\mathrm{SiO}_{2}, \mathrm{Al}_{2} \mathrm{O}_{3}$ or $\mathrm{ZnO}$ is fairly high, which is unfavorable for practical applications. Therefore, MAMs with light weight, small thickness, strong chemical stability, wide absorption frequency range and excellent absorption performance are greatly desired.

As a strictly two-dimensional material, graphene has evoked extensive interests for its abundant physical properties $^{7-9}$. The low dimension and outstanding electronic properties of graphene make it a promising candidate for fabricating novel electronic devices ${ }^{10,11}$. A crucial issue for putting graphene into device application is to understand the physics of metal-graphene interface in the device ${ }^{12,13}$. Theoretic ${ }^{14-16}$ and experimental ${ }^{12,13,17-19}$ studies have shown that the interfacial electronic interaction between metal and graphene would make graphene show some novel magnetic and electric properties. As a result, the investigation of metal/graphene heterostructure is of both fundamental and technological significance. Here we synthesized the graphene-coated Fe nanocomposites $(\mathrm{Fe} / \mathrm{G})$ and investigated their microwave absorption properties. Due to the interfacial coupling between graphene and $\mathrm{Fe}$, the dielectric properties of the nanocomposites are greatly improved, which leads to an excellent microwave absorption performance in a wide frequency range. 


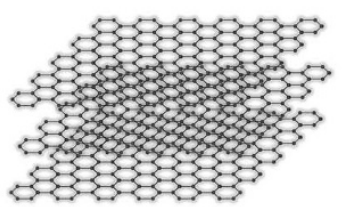

Modified Hummer's Method

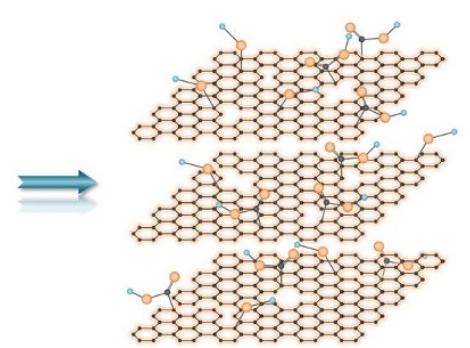

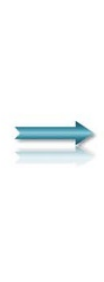

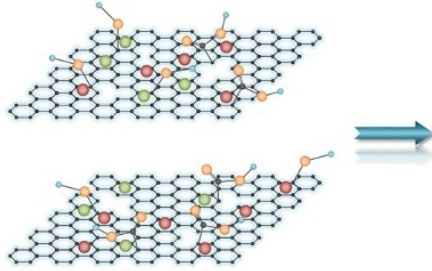

Hydrothermal \& Reduction Reaction

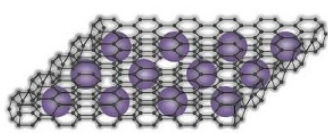

- $\mathrm{C}$

○ $\mathrm{O}$

- $\mathrm{H}$

- $\mathrm{Fe}^{2+}$

- $\mathrm{Fe}^{3+}$

- Fe nanoparticle

Graphite

Graphene Oxide (GO)

Mixed Solution of

Graphene-coated Fe

Nanocomposites

Figure 1 Schematic diagram for the synthetic process of Fe/G nanocomposites.

\section{Results}

The growth of $\mathrm{Fe} / \mathrm{G}$. Fe/G nanocomposites were synthesized by the combination of the hydrothermal and reduction reactions. The detailed process is schematically illustrated in Fig. 1. Graphene oxide (GO) is first prepared from high-purity graphite by modified Hummer's method ${ }^{20}$. After strong oxidation, the hexagonal carbon lattice of graphite is intensively disrupted and a substantial fraction of the carbon network is bonded to functional groups such as $-\mathrm{OH}$ and $-\mathrm{COOH}$, resulting in negatively charged GO sheets which are suspended in the solvent ${ }^{20}$. When ferrous sulfate solution is added to the GO solution, iron ions are absorbed by GO sheets via electrostatic interaction. Under the hydrothermal condition, $\mathrm{GO}$ is reduced to graphene while the iron ions are transformed to $\mathrm{Fe}_{3} \mathrm{O}_{4}$ nanoparticles simultaneously. The intermediate products are then reduced by annealing in hydrogen, leading to the formation of $\mathrm{Fe} /$ $\mathrm{G}$ nanocomposites. In order to compare the microwave absorption properties, a mechanical mixture of $\mathrm{Fe}$ and graphene is prepared which is treated as control group. The mass ratio of $\mathrm{Fe}$ nanoparticle and graphene in the mixture is based on the Energy dispersive spectroscopy (EDS) result of Fe/G, which is shown in Fig. 2b.

Crystal structure. It is well known that Raman technique is particularly useful for identifying the existence of graphene $e^{21}$. Fig. 2a shows the Raman spectrum of Fe/G, in which four peaks of $D$ $\left(\sim 1348 \mathrm{~cm}^{-1}\right), \mathrm{G}\left(\sim 1584 \mathrm{~cm}^{-1}\right), 2 \mathrm{D}\left(\sim 2692 \mathrm{~cm}^{-1}\right)$ and $\mathrm{D}+\mathrm{D}^{\prime}$ $\left(\sim 2943 \mathrm{~cm}^{-1}\right)$ are observed. This Raman result is consistent with that of reduced GO with defects ${ }^{21,22}$. Fig. $2 \mathrm{~b}$ plots the XRD pattern of
$\mathrm{Fe} / \mathrm{G}$, in which all the peaks can be indexed as the $\alpha$-Fe (BCC phase, Im-3m space group) with lattice constant of $a=b=c=2.886 \AA$. Although Fe nanoparticles are easily oxidized in air even at room temperature, there are no peaks of iron oxides in Fig. 2b, which suggests that Fe nanoparticles would be coated by graphene. EDS result of Fe/G (inset of Fig. 2b) shows that no element $\mathrm{O}$ exists in the sample. Here, a small quantity of element $S$ comes from the raw material of ferrous sulfate.

Magnetism and microstructure. The magnetic hysteresis loop for $\mathrm{Fe} / \mathrm{G}$ was measured at room temperature. As shown in Fig. 3, a typical ferromagnetic curve with small coercivity is observed. According to the mass percent of $\mathrm{Fe}$ in $\mathrm{Fe} / \mathrm{G}$, the calculated magnetization of the sample is about $201 \mathrm{emu} \mathrm{g}^{-1}$, which is very close to the saturated magnetization of bulk Fe $\left(217 \mathrm{emu} \mathrm{g}^{-1}\right)$. This result further suggests the magnetic material in the nanocomposite is Fe instead of iron oxide.

In order to investigate the microstructure, the electron microscopic measurement was carried out on the sample. Fig. 4a shows the typical transmission electron microscopy (TEM) image of Fe/G. It is obvious that the Fe nanoparticles inlay in the corrugated graphene sheet and the grain size of Fe nanoparticles ranges from 40 to $100 \mathrm{~nm}$. From the enlarged image shown in the inset of Fig. 4a, a coated structure together with an interface between Fe and graphene are clearly observed. Fig. $4 \mathrm{~b}$ is the scanning electron microscopy (SEM) image of control group, in which a mixture of Fe nanoparticles and graphene without coated structure is observed. As shown in the inset of Fig. 4b, the selected area electron diffraction (SAED)
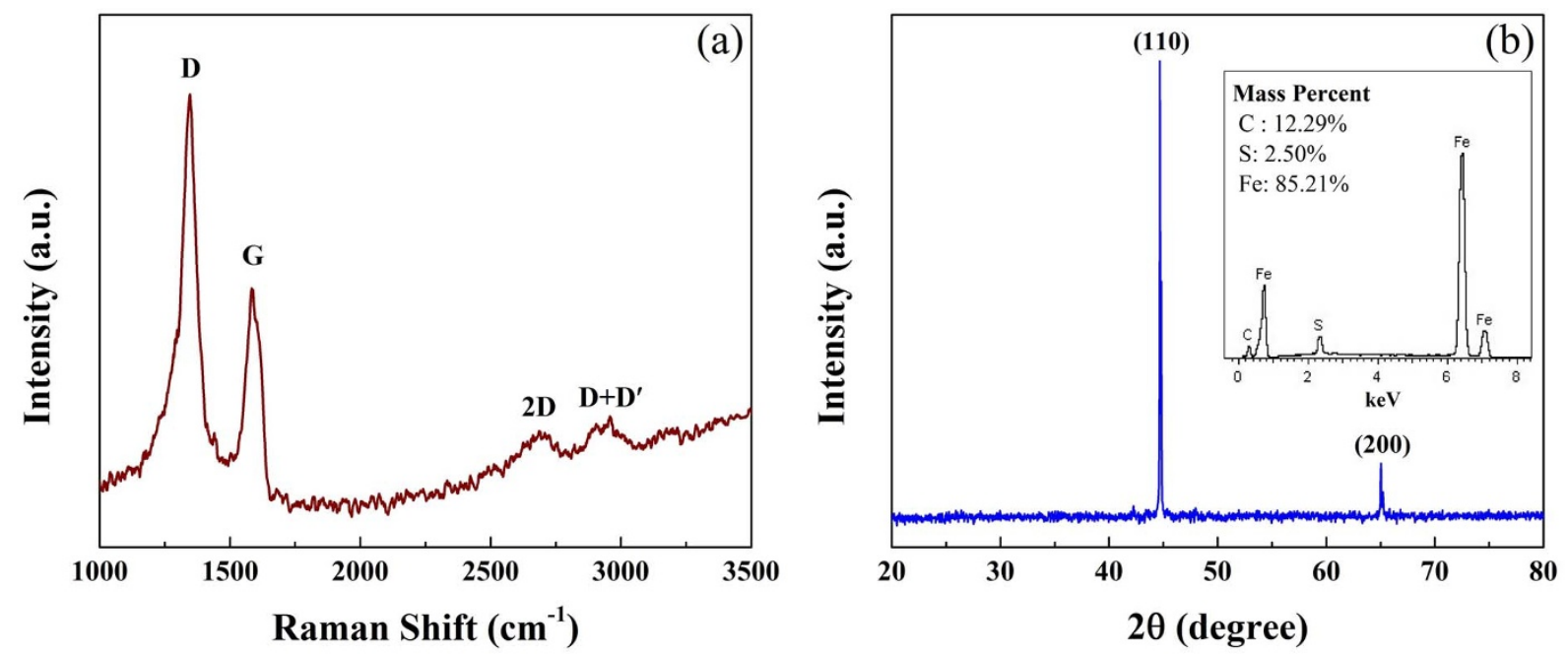

Figure $2 \mid$ (a) Raman Spectrum for Fe/G. (b) XRD pattern for Fe/G. The inset of (b) is the EDS result for Fe/G. 


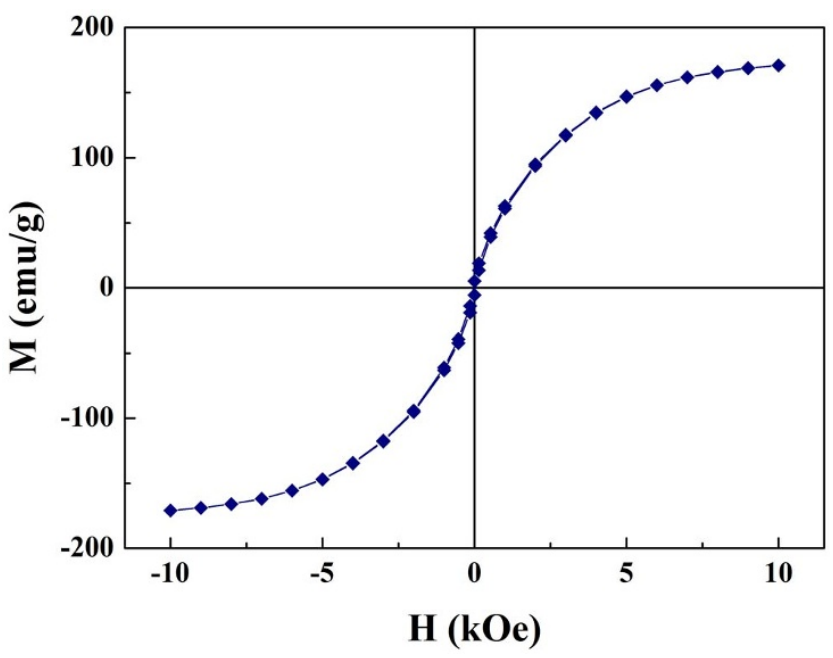

Figure $3 \mid$ The magnetic hysteresis loop for Fe/G at room temperature.

pattern indicates well-defined rings that can be assigned to crystalline bcc-Fe nanoparticles.

Microwave absorption properties. Figure $5 \mathrm{a}$ and $5 \mathrm{~b}$ show the frequency dependence of $\varepsilon_{r}$ and $\mu_{r}$ for the samples in the range of $2 \sim 18 \mathrm{GHz}$, respectively. As shown in Fig. 5a, $\varepsilon_{\text {control }}^{\prime}$ is almost independent on frequency. But in the case of $\mathrm{Fe} / \mathrm{G}$, a largely enhanced $\varepsilon_{\mathrm{Fe} / \mathrm{G}}^{\prime}$ is observed and it decreases with increasing frequency, showing an obvious dielectric dispersion. This phenomenon was seldom observed in other coating structures, such as $\mathrm{Fe} / \mathrm{ZnO}$, $\mathrm{FeNi}_{3} / \mathrm{SiO}_{2}, \mathrm{Fe}_{3} \mathrm{O}_{4} / \mathrm{Fe} / \mathrm{SiO}_{2}$, and $\mathrm{Fe} / \mathrm{C}^{2-4,23}$, which plays a key role in achieving good impedance match. As for the imaginary part $\left(\varepsilon^{\prime \prime}\right)$ of the permittivity (Fig. 5b), the value of $\varepsilon_{\mathrm{Fe} / \mathrm{G}}^{\prime \prime}$ is larger than that of $\varepsilon_{\text {control }}^{\prime \prime}$, indicating an increased dielectric loss. Unlike the dielectric behavior, there are no significant changes of permeability properties between Fe/G and the control group. $\mu_{\mathrm{Fe} / \mathrm{G}}^{\prime}$ and $\mu_{\text {control }}^{\prime}$ show a similar frequency dependence, while the values of $\mu_{\mathrm{Fe} / \mathrm{G}}^{\prime \prime}$ and $\mu_{\text {control }}^{\prime \prime}$ are almost same, indicating a small difference of magnetic loss between FG and the control group.

Based on the data of $\varepsilon_{r}$ and $\mu_{r}$, we calculated the reflection loss (RL) of the samples according to the transmission line theory ${ }^{1}$,

$$
\begin{gathered}
Z_{\text {in }}=\sqrt{\frac{\mu_{r}}{\varepsilon_{r}}} \operatorname{th}\left(\frac{i \cdot 2 \pi f d \sqrt{\mu_{r} \varepsilon_{r}}}{c}\right) \\
R L=20 \lg \left|\frac{Z_{\text {in }}-1}{Z_{\text {in }}+1}\right|(d B)
\end{gathered}
$$

where $Z_{\text {in }}$ is the input impedance of the absorber, $f$ is the frequency of microwave, $d$ is the thickness of the absorber and $c$ is the velocity of light in free space. As illustrated in Fig. 6a, the values of RL for the control group are larger than $-10 \mathrm{~dB}$ with the thickness ranging from 1.5 to $4.0 \mathrm{~mm}$, showing weak microwave absorption ability. However, when $\mathrm{Fe}$ nanoparticles are coated with graphene, the microwave absorption properties of Fe/G nanocomposites are substantially enhanced. For different thickness, the peak values of RL for $\mathrm{Fe} / \mathrm{G}$ are all in the vicinity of $-30 \mathrm{~dB}$ and the minimum $\mathrm{RL}$ is about $-45 \mathrm{~dB}$ at $7.1 \mathrm{GHz}$. It is well known that the effective bandwidth (the frequency range in which RL is less than $-10 \mathrm{~dB}$ ) is another important parameter to evaluate the microwave absorption performance. As shown in Fig. 6b, Fe/G nanocomposites have broad effective bandwidth and it reaches the maximum of $4.4 \mathrm{GHz}$ with the thickness of $2.0 \mathrm{~mm}$, which is larger than that of other Fe-based coated composites ${ }^{2,6,24}$. Comparing with other coating layers, such as $\mathrm{SiO}_{2}$, $\mathrm{Al}_{2} \mathrm{O}_{3}$ or $\mathrm{ZnO}$, the density of graphene is quite low, which makes $\mathrm{Fe} /$ $\mathrm{G}$ a light absorber for microwave absorption.

\section{Discussion}

Generally, there are two key factors for achieving good absorption performance: one is the incident microwave can transmit into the absorbers with minimum reflection; the other is the absorbers can effectively attenuate the transmitted microwave. The former factor is determined by the impedance matching condition while the latter one is related to the intrinsic EM loss ${ }^{1}$. According to the dielectric and magnetic properties of Fe/G and the control group, a distinct change of the relative permittivity is observed when Fe nanoparticles are coated by graphene layer, suggesting that the excellent microwave absorption performance of $\mathrm{Fe} / \mathrm{G}$ is mainly ascribed to the intrinsic change of dielectric properties.

Compared with the control group, there exists an interfacial electronic interaction between $\mathrm{Fe}$ and graphene in $\mathrm{Fe} / \mathrm{G}$, which would affect the electric properties of graphene. According to the recent theoretical results, charges would transfer through the interface in a metal-graphene heterostructure due to their different work function $^{14}$. This theoretical prediction has been demonstrated in a Fe/ graphene $/ \mathrm{SiO}_{2} / \mathrm{Si}$ heterostructure, in which the electron transfer from $\mathrm{Fe}$ to graphene is observed ${ }^{18}$. Therefore, a charge transfer process can be reasonably assumed in the interface of Fe/G, leading to the introduction of free carriers into graphene. The introduced free carriers would vibrate with the stimuli of the microwave and give rise to the electric polarization in graphene, which increases the value of $\varepsilon_{\mathrm{Fe} / \mathrm{G}}^{\prime}{ }^{25}$. Furthermore, $\varepsilon_{\mathrm{Fe} / \mathrm{G}}^{\prime}$ would decrease with the increase of frequency due to the relaxation of polarization, showing a dielectric dispersion $^{25}$. Meanwhile, the motion of the introduced free carriers would attenuate the microwave energy, resulting in the enhancement of the dielectric $\operatorname{loss}^{25}$. In Fe/G, $\varepsilon_{\mathrm{Fe} / \mathrm{G}}^{\prime}$ is markedly increased and shows
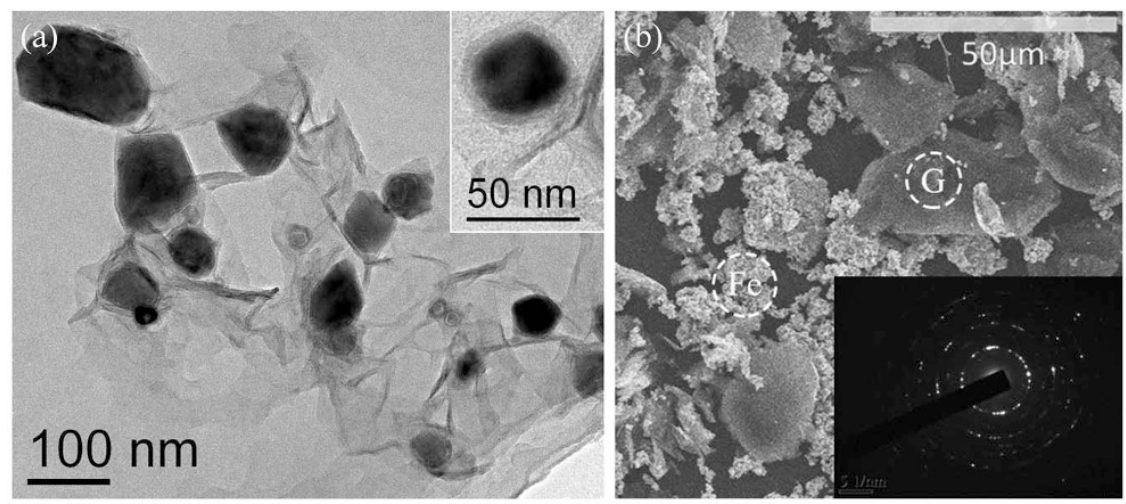

Figure $4 \mid$ (a) TEM image of Fe/G. The inset is an enlarged image of individual Fe nanoparticle, which clearly shows a well-defined coating structure of Fe/G. (b) SEM image of control group. The inset is the SAED pattern of Fe nanoparticle. 

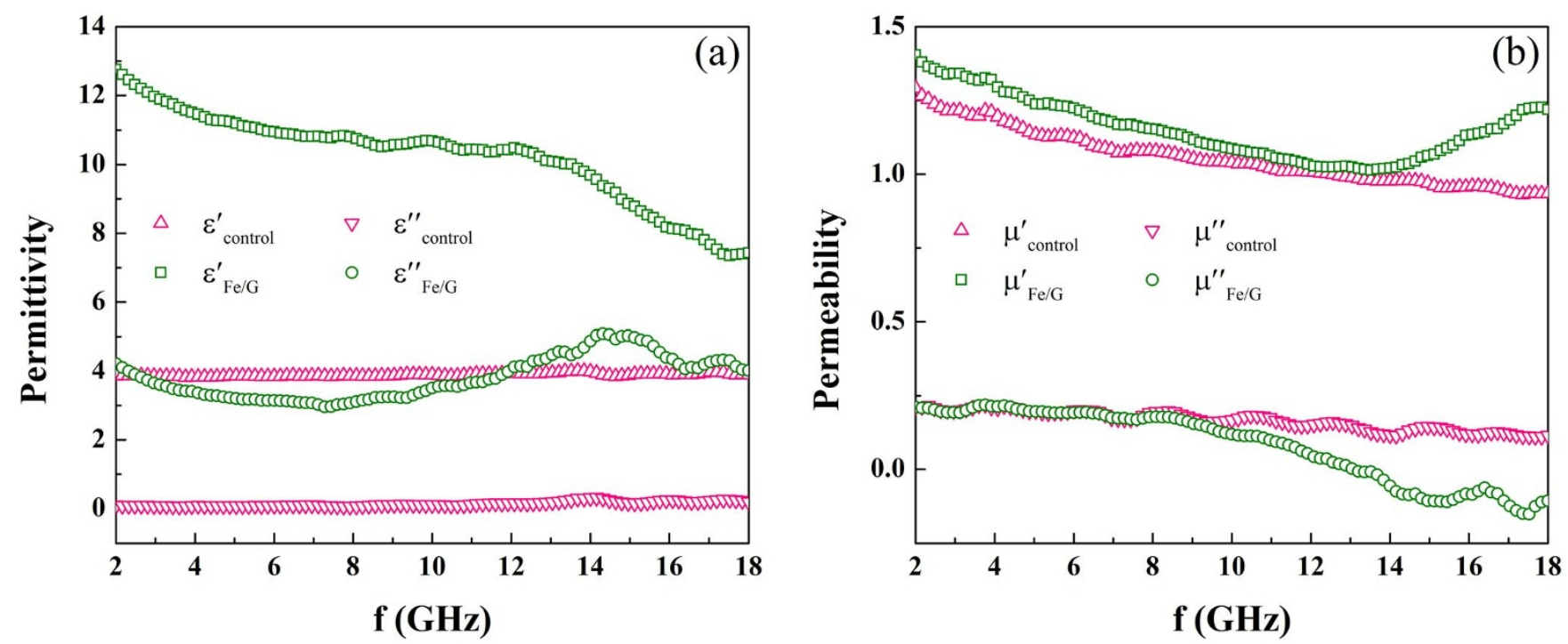

Figure $5 \mid$ Frequency dependence of (a) relative permittivity, and (b) relative permeability for $\mathrm{Fe} / \mathrm{G}$ and the control group.

similar dispersion with $\mu_{\mathrm{Fe} / \mathrm{G}}^{\prime}$, which are helpful to obtain a good impedance matching condition. In addition, the enhanced dielectric loss in $\mathrm{Fe} / \mathrm{G}$ is more favorable for the dissipation of microwave energy. The aforementioned factors would be responsible for the excellent microwave absorption performance of Fe/G. It is reported that the lattice defects in graphene would lead to self-doping, which can also induce some carriers into graphene'. However, this small quantity of carriers in defected graphene would not substantially change the dielectric and microwave absorption properties based on the experimental results of the control group.

In $\mathrm{Fe} / \mathrm{G}$ nanocomposites, there is another mechanism which can also bring about the charge transfer. As we know, the half-filled $p_{z}$ orbitals of graphene are perpendicular to the layer of carbon atoms, forming the bonding $(\pi)$ and antibonding $\left(\pi^{*}\right)$ bands, which touch at a single point (Dirac point) exactly at the corner of the hexagonal Brillouin zone ${ }^{19}$. It has been reported that, in the graphene/Ni(111) heterostructure, the strong hybridization between the graphene $\pi$ and $\mathrm{Ni} 3 \mathrm{~d}$ valence-band gives rise to the charge transfer between $\mathrm{Ni}$ and graphene and leads to some novel electrical and magnetic properties $^{19}$. As for $\mathrm{Fe} / \mathrm{G}, \mathrm{Fe}$ and graphene are synthesized simultaneously and the hybridization of $\mathrm{Fe} 3 \mathrm{~d}$ valence-band and graphene $\pi$ band would occur during the process of co-growth. The charge transfer induced by the strong hybridization would modify the electronic structure of graphene and then change the dielectric property, leading to the excellent microwave absorption performance of Fe/G.

In conclusion, we have developed a catalyst-free method to synthetize the graphene-coated Fe nanocomposites. The dielectric property of this $\mathrm{Fe} /$ graphene coated structure is substantially different from that of uncoated one. Moreover, an excellent microwave absorption performance is observed in Fe/G nanocomposties, which is attributed to the charge transfer at Fe/graphene interface and the polarization of free carriers in graphene. Small RL, broad effective bandwidth, thin thickness, low density, and good chemical stability make Fe/G nanocomposites act as a promising candidate for microwave absorber.

\section{Methods}

Sample preparation. The sample was prepared by hydrothermal and reduction reactions. First, $\mathrm{FeSO}_{4} \cdot 7 \mathrm{H}_{2} \mathrm{O}(6.205 \mathrm{~g})$ was dissolved in distilled water $(75 \mathrm{~mL})$ and $\mathrm{N}_{2} \mathrm{H}_{4} \cdot \mathrm{H}_{2} \mathrm{O}(20 \mathrm{~mL})$ was added into the solution with vigorous stirring. Then, GO solution $\left(15 \mathrm{~mL}, 3 \mathrm{~g} \mathrm{~L}^{-1}\right)$ was diluted by distilled water $(100 \mathrm{~mL})$ and slowly added into the solution. The mixture was then transferred into Teflon-lined stainless steel autoclaves for hydrothermal reaction. After reacting at $130^{\circ} \mathrm{C}$ for $8 \mathrm{~h}$, the resulting black powder was washed by distilled water and alcohol following by annealed in $\mathrm{H}_{2}$ at $600^{\circ} \mathrm{C}$ for $4 \mathrm{~h}$. The final product was labeled as Fe/G. The control group was the
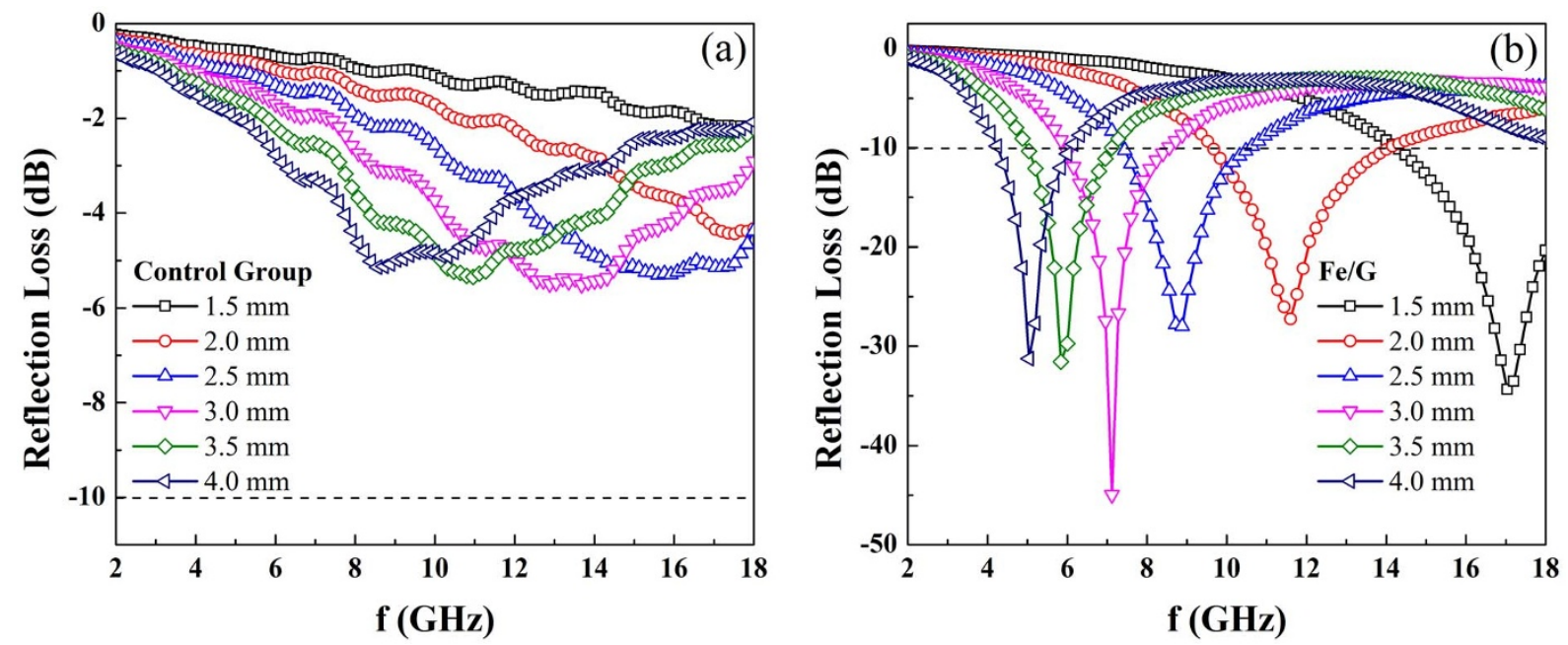

Figure 6 | Calculated reflection loss of different thickness for (a) control group, and (b) Fe/G. The dash line is a guide of eye to show the frequency range in which RL is less than $-10 \mathrm{~dB}$. 
mixture of Fe nanoparticles and graphene, which were individually synthesized by the same procedure and mechanically mixed.

Measurement. The structure of the sample was investigated by power X-ray diffraction (XRD) and Raman spectrum with laser at $514 \mathrm{~nm}$. The microstructure of $\mathrm{Fe} / \mathrm{G}$ was characterized by transmission electron microscope (TEM) and its composition was determined by energy dispersive spectroscopy (EDS). The magnetic measurement was carried out at room temperature by vibrating sample magnetometer (VSM, Lakeshore 7400). The relative permittivity and relative permeability were measured by network analyzer (Agilent PNA E8363B) in the frequency range of $2 \sim 18 \mathrm{GHz}$. For microwave measurement, the samples were prepared by dispersing Fe/G and the control group (40\% wt.) into paraffin matrix (60\% wt.) and then pressing them into hollow cylinders with outer diameter of $7 \mathrm{~mm}$ and inner diameter of $3 \mathrm{~mm}$.

1. Qin, F. \& Peng, H. X. Ferromagnetic microwires enabled multifunctional composite materials. Prog. Mater. Sci. 58, 183-259 (2013).

2. Liu, X. G. et al. Microwave-absorption properties of $\mathrm{ZnO}$-coated iron nanocapsules. Appl. Phys. Lett. 92, 173117-173113 (2008).

3. Yan, S. J. et al. Microwave absorption properties of $\mathrm{FeNi}_{3}$ submicrometre spheres and $\mathrm{SiO}_{2} @ \mathrm{FeNi}_{3}$ core-shell structures. J. Phys. D: Appl. Phys. 43, 245003 (2010).

4. Chen, Y. J. et al. Synthesis, magnetic and electromagnetic wave absorption properties of porous $\mathrm{Fe}_{3} \mathrm{O}_{4} / \mathrm{Fe} / \mathrm{SiO}_{2}$ core/shell nanorods. J. Appl. Phys. 106, 054303-054304 (2009).

5. Longgang, Y.et al. Enhanced microwave absorption of Fe nanoflakes after coating with $\mathrm{SiO}_{2}$ nanoshell. Nanotechnology 21, 095708 (2010).

6. Zhang, Z. D. et al. Microwave absorption properties of $\mathrm{Fe} @ \mathrm{Al}_{2} \mathrm{O}_{3}$ nanoembedments prepared by mechanosynthesis. Mater. Chem. Phys. 130 615-618 (2011).

7. Geim, A. K. \& Novoselov, K. S. The rise of graphene. Nat. Mater. 6, 183-191 (2007).

8. Geim, A. K. Graphene: Status and Prospects. Science 324, 1530-1534 (2009).

9. Castro Neto, A. H., Peres, N. M. R., Novoselov, K. S. \& Geim, A. K. The electronic properties of graphene. Rev. Mod. Phys. 81, 109-162 (2009).

10. Novoselov, K. S. et al. Electric field effect in atomically thin carbon films. Science 306, 666-669 (2004)

11. Schwierz, F. Graphene transistors. Nat. Nanotech. 5, 487-496 (2010).

12. LeeEduardo, J. H. et al. Contact and edge effects in graphene devices. Nat. Nanotech. 3, 486-490 (2008).

13. Huard, B., Stander, N., Sulpizio, J. A. \& Goldhaber-Gordon, D. Evidence of the role of contacts on the observed electron-hole asymmetry in graphene. Phys. Rev. $B$ 78, 121402 (2008)

14. Giovannetti, G. et al. Doping Graphene with Metal Contacts. Phys. Rev. Lett. 101, 026803 (2008)

15. Gong, C. et al. First-principles study of metal-graphene interfaces. J. Appl. Phys. 108, 123711-123718 (2010).
16. Khomyakov, P. A. et al. First-principles study of the interaction and charge transfer between graphene and metals. Phys. Rev. B 79, 195425 (2009).

17. Li, W. et al. Electron localization in metal-decorated graphene. Phys. Rev. B $\mathbf{8 4}$ 045431 (2011).

18. Pi, K. et al. Electronic doping and scattering by transition metals on graphene. Phys. Rev. B 80, 075406 (2009).

19. Yu, S. D. \& Fonin, M. Electronic and magnetic properties of the grapheneferromagnet interface. New J. Phys. 12, 125004 (2010).

20. Park, S. \& Ruoff, R. S. Chemical methods for the production of graphenes. Nat. Nanotech. 4, 217-224 (2009).

21. Ferrari, A. C. \& Basko, D. M. Raman spectroscopy as a versatile tool for studying the properties of graphene. Nat. Nanotech. 8, 235-246 (2013).

22. Xu, H. L., Bi, H. \& Yang, R. B. Enhanced microwave absorption property of bowllike $\mathrm{Fe}_{3} \mathrm{O}_{4}$ hollow spheres/reduced graphene oxide composites. J. Appl. Phys. 111, 07A522-523 (2012).

23. Liu, J. R. et al. Gigahertz range electromagnetic wave absorbers made of amorphous-carbon-based magnetic nanocomposites. J. Appl. Phys. 98, 054305-054307 (2005).

24. Zhang, X. F. et al. Microstructure and microwave absorption properties of carboncoated iron nanocapsules. J. Phys. D: Appl. Phys. 40, 5383 (2007).

25. Toshiyuki, Sameshima., Hiromi, Hayasaka. \& Tomonori, Haba. Analysis of Microwave Absorption Caused by Free Carriers in Silicon. Jpn. J. Appl. Phys. 48, 021204 (2009).

\section{Acknowledgments}

This work is supported by the National Basic Research Program of China (2012CB932304) and National Natural Science Foundation of China (Grant No. U1232210 and 11004095).

\section{Author contributions}

Experiments were designed by X.C.Z. and D.H.W. and carried out by X.C.Z., L.Y.W., K.X. and Y.Y. The data were collected by X.C.Z. and Y.Y. Results were analyzed and interpreted by X.C.Z., Z.M.Z., D.H.W., Y.Y., Q.Q.C. and Y.W.D. The manuscript was written by X.C.Z. and D.H.W. D.H.W. and X.C.Z. is responsible for project direction, planning and infrastructure.

\section{Additional information}

Competing financial interests: The authors declare no competing financial interests.

How to cite this article: Zhao, X.C. et al. Excellent microwave absorption property of Graphene-coated Fe nanocomposites. Sci. Rep. 3, 3421; DOI:10.1038/srep03421 (2013).

(c) (i) (-) $\Theta$ This work is licensed under a Creative Commons Attribution-

cc) visit http://creativecommons.org/licenses/by-nc-nd/3.0 